PROCEEDINGS OF THE

AMERICAN MATHEMATICAL SOCIETY

Volume 132, Number 1, Pages 283-289

S 0002-9939(03)06989-2

Article electronically published on August 19, 2003

\title{
TREE-LIKE CONTINUA AND 2-TO-1 MAPS
}

\author{
JO HEATH AND VAN C. NALL
}

(Communicated by Alan Dow)

\begin{abstract}
It is not known if there is a 2-to-1 map from a continuum onto a tree-like continuum. In fact, it is not known if there is a 2-to-1 map onto a hereditarily decomposable tree-like continuum. We show that the domain of such a map would have to contain an indecomposable continuum.
\end{abstract}

\section{INTRODUCTION}

In 1983 Sam Nadler and Lew Ward [8] asked if a tree-like continuum can be the image of a 2-to-1 map (defined on a continuum, of course). That question is answered only for some cases. For instance, it is known that a tree-like continuum cannot be the image of a 2-to-1 map if it is hereditarily indecomposable [10, or if it is an indecomposable arc-continuum (every proper subcontinuum is an arc) [3]. In the decomposable case, Gottschalk [4] showed that no dendrite can be the image of a 2-to-1 map. Dendrites are uniquely arcwise connected, locally connected continua.

All of the continua listed above, in fact all tree-like continua, are hereditarily unicoherent. Nadler and Ward showed that if a continuum fails to be hereditarily unicoherent, then it is the image of a 2-to- 1 map. Thus in the study of 2-to- 1 images there is no loss of generality if the continuum is assumed to be hereditarily unicoherent. What about the case of continua that are hereditarily unicoherent and yet are not tree-like? For some time it was conjectured that all non-treelike continua, including these hereditarily unicoherent ones, were 2-to-1 images. However, it has been shown [6] that some of Jim Roger's pseudo-solenoids refute this conjecture. This paper considers this conjecture for the hereditarily decomposable case, i.e. is it true that a hereditarily decomposable continuum is the image of a 2-to-1 map iff it is not tree-like? H. Cook [2] has shown that every $\lambda$ dendroid, that is, every hereditarily decomposable and hereditarily unicoherent continuum, is treelike. Cook's result, combined with the result of Nadler and Ward mentioned above, tells us that a hereditarily decomposable continuum that is not tree-like must be the image of a 2-to-1 map from a continuum. Therefore, if it can be shown that there is no 2-to- 1 map from a continuum onto a $\lambda$ dendroid; then the original conjecture restricted to hereditarily decomposable continua would be settled affirmatively.

Received by the editors November 16, 2001 and, in revised form, July 27, 2002.

2000 Mathematics Subject Classification. Primary 54C10.

Key words and phrases. 2-to-1 map, tree-like continuum, dendroid, indecomposable continuum. 
However, it is not yet known if there is a 2-to-1 map from a continuum onto a dendroid (i.e. an arcwise connected $\lambda$ dendroid). Our main result is that no hereditarily decomposable continuum maps 2-to-1 onto a dendroid. This theorem could be greatly strengthened if it were known that there is no at most 2-to-1 map from an indecomposable continuum onto a dendroid, and we raise this question in the last section. On the way to the main theorem we develop structure theorems concerning 2-to-1 maps onto dendroids or $\lambda$ dendroids; for instance, every $\lambda$ dendroid that is the reduced image of a 2-to- 1 map is an infinite-od. (Reduced means that no proper subcontinuum of the image has connected preimage.)

We have included a glossary of terms just before the bibliography.

\section{Structure Results}

Every 2-to-1 map from one continuum to another has a restriction to a subcontinuum of the domain that is 2-to- 1 and reduced. Knowing that every proper subcontinuum of the image has disconnected preimage is a useful hypothesis, and if the original image is tree-like or a dendroid, for instance, then the reduced map is still onto a tree-like continuum or onto a dendroid. In this way, if one assumes the existence of a 2-to-1 map onto a hereditarily decomposable tree-like continuum, then there is no loss in generality if the map is assumed to be reduced. We make this assumption often without discussion.

In the following lemma we extract the parts of [7, Lemma B] that we need. The definition of a c-set is in the glossary.

Lemma 1 (Harlan Cross Miller). If $M$ is a hereditarily decomposable continuum, and $a$ and $b$ are points in $M$ such that $M$ is irreducible from a to $b$, then there is $a$ continuum $K$ in $M \backslash\{a, b\}$ such that $M \backslash K$ is the union of two mutually separated connected sets $U$ and $V$ such that 1) $M=\bar{U} \cup \bar{V}$, and 2) $\bar{U} \cap K$ is a c-set in $\bar{U}$, and $\bar{V} \cap K$ is a c-set in $\bar{V}$.

Lemma 2. Suppose $M, a, b$, and $K$ are as in Lemma 1, and $f$ is a simple map from a continuum onto $M$. Then $f^{-1}(K)$ has at most two components.

Proof. Let $C$ be a component of $f^{-1}(K)$, and let $C_{1}, C_{2}, \ldots$ be a nested sequence of continua containing $C$ properly whose intersection is $C$. If $f\left(C_{i}\right) \cap U \neq \emptyset$, then each component of $f\left(C_{i}\right) \cap U$ runs to the boundary of $f\left(C_{i}\right) \cap U$. That is, the boundary relative to $f\left(C_{i}\right)$. Therefore, there is a component of $f\left(C_{i}\right) \cap \bar{U}$ that contains points in $U$ and $\bar{U} \backslash U$, and $\bar{U} \backslash U$ is contained in $K$. Therefore, $f\left(C_{i}\right)$ contains $K \cap \bar{U}$, since it is a c-set in $\bar{U}$. Similarly if $f\left(C_{i}\right) \cap V \neq \emptyset$, then it contains $K \cap \bar{V}$. It follows that $f(C)$ contains either $\bar{U} \cap K$ or $\bar{V} \cap K$. Since $(\bar{U} \cap K) \cap(\bar{V} \cap K) \neq \emptyset, f^{-1}(K)$ has at most two components.

In the following lemma the notation $\mathcal{C}(A)$ represents the number of components of a set $A$.

Lemma 3. Suppose $f$ is a reduced map from the continuum $X$ onto the continuum $Y$. If $K$ is a proper subcontinuum of $Y$ whose inverse has finitely many components, then $Y \backslash K$ has fewer components than does the inverse of $K$.

Proof. Let $E$ denote the finite collection of components of $f^{-1}(K)$. Then $E$ has at least two elements since $f$ is reduced. There is a component $D_{1}$ of $Y \backslash K$ such that $f^{-1}\left(\bar{D}_{1}\right)$ contains a continuum that intersects two of the elements of $E$, say $C_{1}$ and 
$C_{2}$. If $f^{-1}\left(K \cup \bar{D}_{1}\right)$ is not yet connected, then there is a component $D_{2}$ of $Y \backslash K$ and another element $C_{3}$ of $E$ such that $f^{-1}\left(\bar{D}_{2}\right)$ contains a continuum that intersects $\left(C_{1} \cup C_{2}\right)$ and $C_{3}$. Continuing by induction we have a collection $D_{1}, D_{2}, D_{3}, \ldots, D_{n-1}$ such that $f^{-1}\left(K \cup \bar{D}_{1} \cup \bar{D}_{2} \cup \ldots \cup \bar{D}_{n-1}\right)$ is connected and $f^{-1}(K)$ has at least $n$ components. Therefore, the continuum $K \cup \bar{D}_{1} \cup \bar{D}_{2} \cup \ldots \cup \bar{D}_{n-1}=Y$, since $f$ is reduced. That is, $Y \backslash K$ has at most $n-1$ components.

Corollary 1. Suppose $f$ is a reduced map from the continuum $X$ onto an $\infty$-od $Y$ and $K$ is a subcontinuum of $Y$ whose complement has infinitely many components. Then the inverse of $K$ has infinitely many components.

Lemma 4. If $f$ is a reduced 2-to-1 map from a continuum onto the continuum $M$, then $M$ does not contain a pair of disjoint continua $E$ and $F$ such that $M /\{E, F\}$ is hereditarily decomposable and irreducible from $E$ to $F$.

Proof. If $M$ does contain such a pair of continua, then from Lemma 1 and Lemma 2 there is a continuum $K$ contained in $M \backslash(E \cup F)$ that separates $M$, and such that $f^{-1}(K)$ has at most two components. This contradicts Lemma 3

Theorem 1. If a unicoherent, hereditarily decomposable continuum is the image of a reduced 2-to-1 map from a continuum, then it is an $\infty$-od.

Proof. Assume $Y$ is a unicoherent, hereditarily decomposable continuum that is the image of a 2-to-1 map from a continuum. According to [5] we must produce for each $n$ a continuum $K_{n}$ such that $Y \backslash K_{n}$ has at least $n$ components. Since $Y$ is decomposable and unicoherent, there is a continuum $K_{2}$ that separates $Y$.

Assume there is a continuum $K_{n-1}$ such that $Y \backslash K_{n-1}$ has at least $n-1$ components. If $Y \backslash K_{n-1}$ has infinitely many components, then we are done. So assume the number of components of $Y \backslash K_{n-1}$ is finite. Let $C$ be one of these components. Now $\bar{C}$ is the union of two proper subcontinua $A$ and $B$, and $E=A \cap B$ is a continuum since $Y$ is unicoherent. If $E \cap K_{n-1} \neq \emptyset$, let $K_{n}=K_{n-1} \cup E$.

If $E \cap K_{n-1}=\emptyset$, assume without loss of generality that $B \cap K_{n-1} \neq \emptyset$, and $A \cap K_{n-1}=\emptyset$. By Lemma 4, there is a proper subcontinuum $N$ of $Y$ that contains $A$ and $Y \backslash C$. If $K_{n}=K_{n-1} \cup(N \cap C) \cup E$, then $Y-K_{n}$ has at least $n$ components.

Corollary 2. If a $\lambda$-dendroid does not contain an $\infty$-od, then it is not the image of a 2-to-1 map from a continuum.

\section{The MAIN RESUlTS}

Now we must review a couple of earlier results about dendroids. In [1 Borsuk showed that in a dendroid the closure of the union of a nested collection of arcs is an arc. Also in [9] Smithson showed that if a continuum has this nested arc property, then it is not the image of a 2-to-1 map from an arc-connected continuum. However, Smithson's proof relies on a slightly weaker assumption which we state in the following lemma.

Lemma 5. There does not exist a 2-to-1 map from a continuum onto a dendroid such that the preimage of each point in the range is contained in an arc in the domain.

Lemma 6. If $f$ is a simple map from a hereditarily decomposable continuum $X$ onto a dendroid $D$, and $a$ and $b$ are distinct points of $X$ such that $f(a)=f(b)$, then there is an arc in $X$ that contains $a$ and $b$. 
Proof. Suppose there is a point $y$ of $D$ such that no $\operatorname{arc}$ in $X$ contains the two inverse points, $a$ and $b$, of $y$.

Consider the collection of triples of the form $(M, A, B)$ where $M$ is a continuum in $X,(A, B)$ is a crisp pair of arcs in $X$ (meaning that they are disjoint arcs and $f$ restricted to each is a homeomorphism onto the same arc in $D), a$ is an endpoint of $A, b$ is an endpoint of $B$, and either

Case (1). $A \cap M=\left\{a^{\prime}\right\}$, where $a^{\prime}$ is the other endpoint of $A$, and either $B \cap M=\left\{b^{\prime}\right\}$, where $b^{\prime}$ is the other endpoint of $B$, or $B \cap M$ is a subarc of $B$ containing $b^{\prime}$; or

Case (2). $B \cap M=\left\{b^{\prime}\right\}$ and either $A \cap M=\left\{a^{\prime}\right\}$ or $A \cap M$ is a subarc of $A$ containing $a^{\prime}$.

We say that $\left(M_{1}, A_{1}, B_{1}\right)<\left(M_{2}, A_{2}, B_{2}\right)$ if $M_{2}$ is a proper subcontinuum of $M_{1}$, $A_{1}$ is a proper subarc of $A_{2}$, and $B_{1}$ is a proper subarc of $B_{2}$. (So the continua shrink as the arcs elongate.)

Claim 1. The collection is non-empty.

Let $I$ be a subcontinuum of $X$ irreducible from $a$ to $b$ and decompose $I$ into $H \cup K$, two proper subcontinua. One, say $H$, must contain $a$ and the other $b$ and neither $a$ nor $b$ is in the intersection $H \cap K$. Let $\lambda$ be the arc in $D$ from $y$ to $f(H \cap K)$, so that only its endpoint $p$ is in $f(H \cap K)$. Denote by $q$ a point in $H \cap K$ that maps to $p$. Then, since $a$ maps to $y, q$ maps to $p$ and $\{a, q\}$ is a subset of $H$, $f(H)$ contains the $\operatorname{arc} \lambda$. We use here and later that dendroids are uniquely arcwise connected. Also, $f(K)$ contains $\lambda$. Let $\left\{p_{i}\right\}$ be a sequence of points in $\lambda$ converging to $p$ (but none equal to $p$ ). Since the arc $\left\langle y, p_{i}\right\rangle$ is contained in $f(H \backslash K) \cap f(K \backslash H)$ for each $i$, and since $f$ is simple, there is an arc $A_{i}$ in $H \backslash K$ such that $f \mid A_{i}$ is a homeomorphism onto $\left\langle y, p_{i}\right\rangle$. (Also, a similar arc exists in $K \backslash H$.) Let $U=\bigcup_{i} A_{i}$. Since $\bar{U} \backslash U$ maps to $p$ and is a continuum, $\bar{U} \backslash U$ is a single point that maps to $p$ and $\bar{U}$ is an arc $A$ that lies in $H \backslash K$, except possibly for the endpoint that maps to $p$. The same thing happens in $K \backslash H$ and these endpoints that map to $p$ cannot be the same point (or else the inverse of $y$ is contained in an arc). Let $B$ be the similar arc that is in $K \backslash H$ except possibly for its endpoint that maps to $p$. Note that $(A, B)$ is a crisp pair of arcs.

One of $A$ or $B$ has as its endpoint the point $q$ in $H \cap K$. If it is $A$, we have Case (1). If it is $B$, we have Case (2). Suppose it is $A$. Since $p$ is the only point of $\lambda$ in $f(H \cap K)$, the arc $A$ lies outside of $K$ except for its endpoint $q$, so if $K$ is used as our continuum $M$ in the construction of a triple, then $A \cap M$ is a single point and the $\operatorname{arc} B$ is entirely inside $M$.

Claim 2. Each chain in the collection has an upper bound.

Suppose $\left\{\left(M_{i}, A_{i}, B_{i}\right)\right\}$ is an ascending chain from the collection of triples. Let $M=\bigcap_{i} M_{i}$, and let $A$ and $B$ be the closures of $\bigcup_{i} A_{i}$ and $\bigcup_{i} B_{i}$, respectively. The nesting property of $D$ and our assumption on $y$ ensure that these closures add but a single point $a^{\prime}$ to $\bigcup_{i} A_{i}$ and a single point $b^{\prime}$ to $\bigcup_{i} B_{i}$, and these are distinct points that map to the same point in $D$. So $(A, B)$ is a crisp pair of $\operatorname{arcs}$ since $\left(A_{i}, B_{i}\right)$ is a crisp pairs of arcs for each $i$.

Subclaim. If, for infinitely many $i$, Case (1) holds for the triple $\left(M_{i}, A_{i}, B_{i}\right)$, then Case (1) holds for $(M, A, B)$.

Since $A_{i} \cap M_{i}=\left\{a_{i}\right\}$ for infinitely many $i$, the union of these $A_{i}$ (which equals the union of all of them) does not intersect $M$, and since $A$ adds only $a^{\prime}$ to the union and $a^{\prime}$ is in $M$, we have that $M \cap A=\left\{a^{\prime}\right\}$. 
If $B_{i} \cap M_{i}$ is a single point for infinitely many $i$, then we are done since $M \cap B=$ $\left\{b^{\prime}\right\}$ satisfies Case (1). So, suppose without loss of generality that for every integer $i$, the intersection $B_{i} \cap M_{i}$ is a subarc of $B_{i}$, one of whose endpoints is $b_{i}$, the endpoint of $B_{i}$ that is not $b$. We only need that $M \cap B$ is connected, since Case (1) allows this intersection to be the point $b^{\prime}$ or an $\operatorname{arc}$ in $B$ containing $b^{\prime}$, and we know that $b^{\prime}$ is in both $B$ and $M$.

Suppose $B \cap M$ is not an endarc of $B$ (or just the point $b^{\prime}$ ). Let $z$ be the first point of $B$ (in the order from $b$ to $b^{\prime}$ ) that lies in $M$. Since $B \cap M$ is not an endarc, there is a point $w \in B \backslash M$ between $z$ and $b^{\prime}$. Since $b^{\prime}$ is the only point of $B$ that is not in $\bigcup_{i} B_{i}$, there is a $j$ such that $w$ is in $B_{j}$. If $w$ is not in $M_{j}$, then $z$ is not either, since $B_{j} \cap M_{j}$ is an endarc of $B_{j}$ and $z$ comes before $w$. But $z$ not in $M_{j}$ means $z$ is not in $M$. This is a contradiction and we see that if $w$ is in any $B_{i}$, then $w$ must be in $M_{i}$. But $w \in B_{j}$ implies that $w \in B_{k}$ for every $k>j$, since the arcs are ascending. Therefore $w \in M_{k}$ for all $k>j$. This means that $w$ is in $M$. Another contradiction.

Let $(M, A, B)$ be a maximal triple. Without loss of generality we will assume that $(M, A, B)$ is a Case (1) triple and that $B \cap M$ is an arc rather than just a point. Let $I$ be an irreducible continuum in $M$ from $a^{\prime}$ (the other endpoint of $A$, the one in $M)$ to the $\operatorname{arc} B \cap M$.

We need to see that $B \cap I$ is connected and contains $b^{\prime}$, the endpoint of $B$ that is not $b$. It is non-empty since $I$ is irreducible from $a^{\prime}$ to $B$, so let $z$ be the first point of $B$ (in the order from $b$ to $b^{\prime}$ ) that lies in $I$, and denote by $\gamma$ the subarc $\left\langle z, b^{\prime}\right\rangle$ of $B$. Now, suppose there is a point $w$ of $\gamma$, excluding $b^{\prime}$, that is not in $I$. Note that it is not possible for $b^{\prime}$ to be the only point of $\gamma$ that is not in $I$. Consider the inverse of $f(w)$. One point, $w$, of $B \backslash I$ maps to $f(w)$; another point, $w^{\prime}$, of $A$ maps to $f(w)$, where $w^{\prime}$ lies in $A \backslash I$ since only the point $a^{\prime}$ of $A$ lies in $M$ or in $I$; and yet, because both $z$ and $a^{\prime}$ are in $I$, a third point, a point in $I$, maps to $f(w)$ since both $\gamma$ and $I$ map onto the (only) arc from $f(z)$ to $f\left(a^{\prime}\right)=f\left(b^{\prime}\right)$. This contradicts the fact that $f$ is simple.

Decompose $I$ into $H \cup K$, two proper subcontinua. One, say $H$, will contain $a^{\prime}$, $K$ will contain the endarc $\gamma$ of $B$, and neither $\gamma$ nor $a^{\prime}$ will intersect $H \cap K$ since $I$ is irreducible from $a^{\prime}$ to $M \cap B$.

Recall that $a^{\prime}$ and $b^{\prime}$ map to the same point, say $y^{\prime}$, in $D$. Let $\lambda$ be the arc in $D$ from $y^{\prime}$ to $f(H \cap K)$, so that only its endpoint $p$ is in $f(H \cap K)$. As before, in the proof of Claim 1, we get a crisp pair of arcs, say $(\alpha, \beta)$, from each of $a^{\prime}$ and $b^{\prime}$, respectively, to points $q$ and $s$ that map to $p$, where $q$ is in $H \cap K$. Here is where one of the special properties of the triples is used: the arc $\alpha$ from $a^{\prime}$ to either $q$ or $s$ lies in $M$ and the arc $A$ from $a$ to $a^{\prime}$ lies outside of $M$, except for $a^{\prime}$ only, so their union, say $A^{\prime}$, is an elongated arc and not some other continuum. We need to see now that the other continuum $B \cup \beta=B^{\prime}$ is also an arc and that $\left(A^{\prime}, B^{\prime}\right)$ is a crisp pair of arcs. Each of $A$ and $B$ maps onto $f(A)$ since $(A, B)$ is a crisp pair, and so, since $f$ is simple, there are no other points that map to $f(A)$. So $f(\alpha)$ is disjoint from $f(A)$ except for $y^{\prime}$, and $f(\beta)$ maps onto $f(\alpha)$, so no point of $\beta$ is in $B$, except for their common endpoint, $b^{\prime}$. Thus $B^{\prime}$ is an arc and $\left(A^{\prime}, B^{\prime}\right)$ is a crisp pair of arcs.

The arc $\lambda$ from $y^{\prime}$ to $p$ has only the point $p$ in $f(H \cap K)$, so one of $A^{\prime}$ or $B^{\prime}$, whichever contains $q$, intersects $H \cap K$ exactly in the one point $q$. If $q$ is in $A^{\prime}$, then 
the larger triple is $\left(K, A^{\prime}, B^{\prime}\right)$. If $q$ is in $B^{\prime}$, then the larger triple is $\left(H, A^{\prime}, B^{\prime}\right)$. Thus the triple $(M, A, B)$ was not maximal, a contradiction.

The following theorem follows easily from the previous two lemmas.

Theorem 2. There does not exist a 2-to-1 map from a hereditarily decomposable continuum onto a dendroid.

Question. Is there an indecomposable continuum that admits a simple map onto a dendroid?

\section{Glossary}

(1) A topological space is a continuum if it is compact, connected and metrizable.

(2) A 2-to-1 map is crisp if the preimage of each proper subcontinuum in the image consists of two components, each of which maps homeomorphically onto the subcontinuum.

(3) If $M$ is a continuum, a subcontinuum of $N$ of $M$ is a $c$-set in $M$ provided $N$ is a subset of any subcontinuum of $M$ that contains both a point in $N$ and a point not in $N$.

(4) A continuum is a dendrite if it is hereditarily decomposable, hereditarily unicoherent, arc connected, and locally connected.

(5) A continuum is a dendroid if it is hereditarily decomposable, hereditarily unicoherent, and arc connected.

(6) A continuum is an $\infty$-od if it contains a subcontinuum whose complement has infinitely many components.

(7) A continuum is a $\lambda$ dendroid if it is hereditarily decomposable and hereditarily unicoherent.

(8) A map between continua is reduced if no proper subcontinuum of the image has connected preimage.

(9) A map is simple if the inverse of each point in the range contains at most two points.

\section{REFERENCES}

[1] K. Borsuk, A theorem on fixed points, Bull. Acad. Polon. Sci. Cl. III. 2 (1954) 17-20. MR 16:275h

[2] H. Cook, Tree-likeness of dendroids and $\lambda$ dendroids, Fund. Math. 68 (1970) 19-22. MR 41:6171

[3] W. Debski, J. Heath, and J. Mioduszewski, Exactly two-to-one maps from continua onto some tree-like continua, Fund. Math. 141 (1992) 269-276. MR 94b:54093

[4] W. H. Gottschalk, On k-to-1 transformations, Bull. Amer. Math. Soc. 53 (1947), 168-169. MR 8:481d

[5] J. Heath, On n-ods, Houston J. Math. 9 (1983), 477-487. MR 85f:54068

[6] J. Heath, A non-treelike continuum that is not the 2-to-1 image of any continuum, Proc. Amer. Math. Soc. 124 (1996), no. 11, 3571-3578. MR 97c:54017

[7] H. C. Miller, On unicoherent continua, Trans. Amer. Math. Soc. 69 (1950), 179-194. MR 12: $118 \mathrm{~h}$

[8] S. B. Nadler, Jr. and L. E. Ward, Jr., Concerning exactly $(n, 1)$ images of continua, Proc. Amer. Math. Soc. 87 (1983), 351-354. MR 84c:54059 
[9] R. E. Smithson, On the existence of exactly $(2,1)$ maps, Proc. Amer. Math. Soc. 99 (1987), 577-580. MR 88a:54075

[10] J. Heath, 2-to-1 maps with hereditarily indecomposable images., Proc. Amer. Math. Soc. 113 (1991), no. 3, 839-846. MR 92c:54012

Department of Mathematics, Auburn University, Auburn, Alabama 36849

E-mail address: heathjw@auburn.edu

Department of Mathematics \& Computer Science, University of Richmond, Richmond, VIRGINIA 23173

E-mail address: vnall@richmond.edu 